\title{
Seguridad alimentaria nutricional según percepción en hogares de Kukra Hill, Región Autónoma de la Costa Caribe Sur, Nicaragua 2015
}

\section{Food and Nutrition Security as perceived by families of Kukra Hill, Autonomous Region of South Caribbean Coast, Nicaragua, 2015}

Alice Marie Pineda Whitaker

Profesor Titular I, Docente-Investigador,UNAN Managua

a_alicia64@yahoo.com

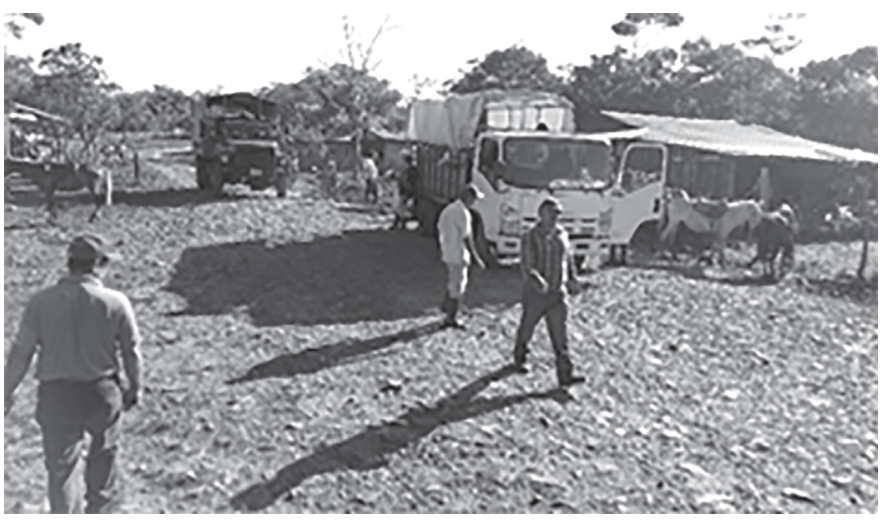

\section{RESUMEN}

El estudio tiene como objetivo conocer la situación de Seguridad Alimentaria Nutricional y algunos factores asociados, en los hogares del municipio de Kukra Hill-Región Autónoma Costa Caribe Sur (RACCS). Es un estudio analítico de factores de riesgo, con una muestra constituida por 107 jefes de hogares. El instrumento utilizado fue la Escala Latinoamericana y Caribeña de Seguridad Alimentaria, se agregaron algunos indicadores condicionantes. Entre los principales hallazgos se destaca que la Inseguridad Alimentaria afecta a la tercera parte de los hogares estudiados. La inseguridad Alimentaria Severa alcanzo un 32\%. Los resultados de la encuesta evidencia preocupación en más de la mitad de los hogares de que el alimento se acabara, y por falta de dinero u otros recursos veían la disponibilidad y el acceso limitado. Más de la mitad de los hogares no tienen una alimentación nutritiva, hay poca variedad de alimentos. En relación a la cantidad de alimentos, el 50\% de los hogares en algún momento se quedaron sin alimento, muchos comieron menos de lo acostumbrado y hasta dejaron de desayunar, almorzar o cenar, por falta de dinero u otro recurso. El hambre afecto a todos los hogares, donde algunos de los integrantes, sintieron hambre pero no había que comer, por lo tanto no comieron y a veces solo comieron una vez al día, o pasaron todo el día sin comer. La Inseguridad Alimentaria en los hogares de los encuestados estuvo asociado a: ubicación geográfica. Vivir en las zonas $3, \mathrm{OR}=3.43 ; \mathrm{Ji}^{2}=5.03$ vivir en la zona $5, \mathrm{OR}=3.48 ; \mathrm{Ji}^{2}=5.64$. Escolaridad, Ser analfabeto $\mathrm{OR}=2.73 ; \mathrm{Ji}^{2}=3.49$. Ingreso mensual menor a $\mathrm{C} \$ 2,850 \mathrm{OR}=4.72$; $\mathrm{Ji}^{2}=4.30$. Ser de la Etnia Miskita $\mathrm{OR}=4.68 ; \mathrm{Ji}^{2}=5.79$. Cobertura del Ingreso, cubre menos que los gastos mínimos. $\mathrm{OR}=2.05, \mathrm{Ji}=3.48$. Palabras clave: seguridad Alimentaria Nutricional -Cantidad- Calidad-Hambre-Costa Caribe Sur

Recibido: 3 de febrero 2016

Aceptado: 23 de mayo 2016
This study aims to determine the situation of food and nutritional security and some associated factors in households in the municipality of Kukra Hill-South Caribbean Coast Autonomous Region (RACCS). It is an analytical study of risk factors, with a sample consisting of 107 heads of households. The instrument used was the Latin American and Caribbean Food Security Scale, some additional indicators were added. Among the key findings it highlighted that food insecurity affects a third of the households surveyed. Severe food insecurity reached $32 \%$. There was concern in more than half of households that food would ran out, and lack of money or other resources saw availability and access limited. More than half of households do not have a nutritious diet, there is little variety of food. In relation to the amount of food, half of households at some point ran out of food, many ate less than usual and even stopped eating breakfast, lunch or dinner, for lack of money or other resources. Hunger affected all households, where some of the members, were hungry but had nothing to eat, so they did not eat and sometimes ate only once a day, and some spent the entire day without eating. Food Insecurity in the homes of respondents was associated with geographical location. Living in zones $3, \mathrm{OR}=3.43 ;=5.03 \mathrm{Chi}^{2}$ live in zone 5 , $\mathrm{OR}=3.48 ; \mathrm{Chi}^{2}=5.64$. Education, be illiterate $\mathrm{OR}=2.73 ; \mathrm{Chi}^{2}$ $=3.49$. Less than C $\$ 2.850$ monthly income $\mathrm{OR}=4.72 ; \mathrm{Chi}^{2}=$ 4.30. Ethnicity be the Miskito $\mathrm{OR}=4.68 ; \mathrm{Chi}^{2}=5.79$. Income coverage, covers less than the minimum expenses. $\mathrm{OR}=2.05$, $\mathrm{Ji}=3 . \mathrm{Ji}=3$.

Keywords: Nutritional Food Safety -Quantity- Quality- HungerSouth Caribbean Coast 
$\mathrm{H}$ ay seguridad alimentaria cuando todas las personas tienen en todo momento acceso físico, social $\mathrm{y}$ económico a suficientes alimentos inocuos y nutritivos para satisfacer sus necesidades alimenticias y sus preferencias en cuanto a los alimentos a fin de llevar una vida activa y sana. Los cuatro pilares de la seguridad alimentaria son la disponibilidad, el acceso, la utilización y la estabilidad. La dimensión nutricional es parte integrante del concepto de seguridad alimentaria y del trabajo del Comité de Seguridad Alimentaria (CSA, 2009).

Nicaragua tiene $130,334.54 \mathrm{~km}^{2}$ de extensión territorial. Está dividido en 2 partes, la región autónoma costa caribe Norte y la región autónoma costa caribe sur, la costa caribe ocupa el $50 \%$ de la superficie total del país, con una población de 620,640 habitantes según el censo de población y vivienda del año 2005. La región autónoma costa caribe sur tiene una población de 306, 510 hab. Una densidad poblacional de 11.2 habitantes por $\mathrm{km}^{2}$. Fueron constituidos en 1987 conforme a la Ley $N^{\circ} 28$, Estatuto de autonomía de las regiones de la costa atlántica, asimismo la ley de lenguas $\mathrm{N}^{\circ} 162$, y la Ley $\mathrm{N}^{\circ} 445$, reconocen el derecho de los pueblos indígenas y comunidades étnicas en la región, quienes tradicionalmente conviven en el territorio mucho antes de la colonización europea en 1492. La Región Autónoma Costa Caribe Sur comprende los municipios de: Paiwas, La Cruz del Rio Grande, Laguna de Perlas, El Tortuguero, El Rama, El Ayote, Muelle de los Bueyes, Kukra Hill, Corn Island, Bluefields, Nueva Guinea.

El municipio de Kukra Hill pertenece a la Región Autónoma Costa Caribe Sur, localizado en el sector central de la región, a $30 \mathrm{Km}$. al norte de la ciudad de Bluefields. La cabecera municipal está situada a $415 \mathrm{~km}$. de Managua, capital de la República de Nicaragua. El vocablo "Kukra" es pronunciado "Kukara" por los locales. El Municipio tiene una extensión territorial de $1262 \mathrm{~km}^{2}$. Cuenta con una población estimada de 9,744 habitantes, de los cuales el 71\% corresponden a la zona rural y el $29 \%$ a la urbana. En cuanto a la distribución por sexo el $52 \%$ son femeninos y el $48 \%$ masculinos. La distribución étnica de la población: Mestizos el $56 \%$ y $39 \%$ de Creole y 5\% Miskito. Se habla el idioma Español y el Ingles Creole y Miskito. El Municipio de Kukra Hill cuenta con un total de 61 comunidades, divididos en 10 sectores según el Modelo de Salud de la Región (MASIRAAS), pero que a la vez se divide en 8 Rutas para fines de atención a la población priorizadas a través de Visitas Integrales (Brigadas Médicas Móviles).

Según el informe del INIDE (2008), el municipio de Kukra Hill tiene un $69.3 \%$ de pobres extremos en la población, $24.5 \%$ son pobres no extremos y un $6.1 \%$ son no pobres, categoría fundamentada en el método de las N.B.I necesidades básicas insatisfechas. La Seguridad Alimentaria nutricional es un tema priorizado por todos los gobiernos, ya que se ubica en el primer Objetivo de Desarrollo del Milenio como es la reducción del hambre y la pobreza. Los hogares tienen seguridad alimentaria cuando todo el año disponen de acceso a la cantidad y variedad de alimentos inocuos que sus integrantes requieren para llevar una vida activa y saludable. En el hogar, la seguridad alimentaria se refiere a la capacidad de garantizar la disponibilidad de alimentos, ya sea que la familia los produzca o los compre, a fin de satisfacer las necesidades de todos sus integrantes.

La situación nutricional de cada miembro del hogar depende del cumplimiento de diversos requisitos: los alimentos disponibles en el hogar deben distribuirse conforme a las necesidades individuales de sus integrantes; los alimentos deben tener la variedad, calidad e inocuidad suficientes; y cada miembro de la familia debe gozar de buena salud para aprovechar los alimentos consumidos.

El hambre es una causa de la pobreza que impide el acceso a los alimentos, por falta de dinero u otros recursos. Afecta especialmente el desarrollo físico e intelectual del niño y niñas, limita la capacidad de las personas como la posibilidad de producir y generar ingresos para la familia y para el país. Es un tema de Protección Social (pobreza, trabajo, alimento, vivienda, tierra, mercado, manejo ambiental). Se elige la Costa Caribe Sur, región Autónoma, con una riqueza invaluable de la población por ser multiétnica, multicultural, con extrema pobreza, hasta el $69 \%$ de la población, y porque existe en este territorio una historia de concesiones extranjeras y de abandono (INIDE, 2008).

La investigación analiza la Seguridad Alimentaria desde la perspectiva del hogar, a través de la disponibilidad de alimento, el acceso, consumo, expresado a través de la cantidad de alimentos en el hogar, la calidad, variedad y la presencia del hambre. También analiza algunos factores asociados a esta situación, a fin de proponer pautas para establecer políticas públicas municipales que promocionan la igualdad de derechos a la alimentación, a superar la pobreza y el hambre, a mejorar la calidad de vida de las personas que viven en este municipio, a fin de lograr un desarrollo sustentable del municipio.

\section{MATERIALES Y MÉTODOS}

El estudio es descriptivo de corte transversal, con un enfoque cuali-cuantitativo. El universo de estudio lo conformaron los hogares ubicados en el área semi-urbana del municipio de Kukra Hill, Un total de 598 hogares. El área semi-urbano está dividida en 5 zonas de las cuales la zona 1 y 2 son de pobreza alta, la zona 3 de pobreza media y las zonas 4 y 5 de pobreza severa. La estratificación fue por niveles de pobreza. Para determinar el tamaño de la muestra se aplicó la fórmula de Mounch Galindo para poblaciones finitas, y se obtuvo una muestra con un $95 \%$ de confianza y un 5\% de error muestral a partir de la población de 598 hogares, obteniendo un total de 106 hogares a encuestar. Estas se distribuyeron de la siguiente manera: zona I y II de pobreza alta con 58, zona III 
de pobreza media con 15 , zona IV y V de pobreza severa con 33. El procesamiento de la información se hizo a través del programa estadístico Epi-Info Versión 7 y las tablas y gráficos en el programa Excel.

Para determinar la Inseguridad Alimentaria en el hogar, se aplicó la encuesta ELCSA (Escala Latinoamericana y Caribeña de Seguridad Alimentaria). Las preguntas definidas en la escala se relaciona a la preocupación de que los alimentos se acaben en el hogar, la cantidad y calidad de alimentos disponibles en el hogar, y cuando no hay que estrategias utilizan para enfrentarla. Consta de 15 preguntas, de los cuales de la (P1-P8) son las situaciones de inseguridad alimentaria que atraviesan los adultos en el hogar, y una segunda sección (P9- P15) con preguntas referidas a condiciones que afectan a los menores de 18 años en el hogar. Utilizar esta escala va más allá de la percepción de los jefes de hogar, se refieren a situaciones objetivas auto reportadas que los integrantes del hogar experimentan, tales como la reducción de la cantidad de alimentos servidos, la omisión de alguna de las comidas diarias, la presencia de hambre en alguno de los integrantes del hogar, y la suspensión de comidas debido a la falta de dinero $u$ otros recursos. Se agregaron preguntas condicionantes al ELCSA. La encuesta fue realizada con los jefes de hogares.

Esta escala es una medición directa y necesaria para comprender el fenómeno de la seguridad alimentaria en el hogar, de bajo costo, cuyo instrumento ha demostrado validez interna y externa, mide los grados de severidad de la inseguridad alimentaria (leve, moderada, severa) es un instrumento confiable que permite optimizar y focalizar alertas tempranas en políticas de prevención.

Análisis de datos. El análisis estadístico se realizó con Epi-Info versión 7. Se determinó el OR para determinar la relación de la escala con otras variables asociadas con el fenómeno de la Inseguridad Alimentaria. Luego para verificar que esa asociación esta estadísticamente significativa se determinó el Ji cuadrado. Se tomaron en cuenta como factores asociados todas aquellas variables cuyo OR era mayor que 1 y cuyo Ji cuadrado estuvo mayor que 3.45

Consentimiento Informado: Los jefes de los hogares participantes firmaron el consentimiento informado, el cual incluyó los principios éticos para las investigaciones médicas en seres humanos de la declaración de Helsinki.

\section{RESULTADOS y DISCUSION}

En el $67 \%$ de los hogares visitados donde hay mayores de 18 años encontramos inseguridad alimentaria, y en el 58\% de los hogares donde hay menores de 18 años se encontró inseguridad alimentaria. Los hogares donde hay mayores de 18 años el 25\% con Inseguridad Leve, el 13\% de Inseguridad Moderada y el 30\% con Inseguridad Severa y un nivel de
Seguridad Alimentaria del 32\%. En hogares con menores de 18 años de edad, se encontró el 12\% con Inseguridad Leve, el 15\% con Inseguridad Moderada y el 32\% con Inseguridad Severa, y un nivel de seguridad Alimentaria del 42\%. El 53\% de los hogares ubicados en las zonas 3 y 5 sufren de Inseguridad Alimentaria severa, y el 33\% de los hogares que habitan en la zona uno. La zona cuatro presenta un $12 \%$ de inseguridad moderada y el 6\% con Inseguridad Alimentaria severa.

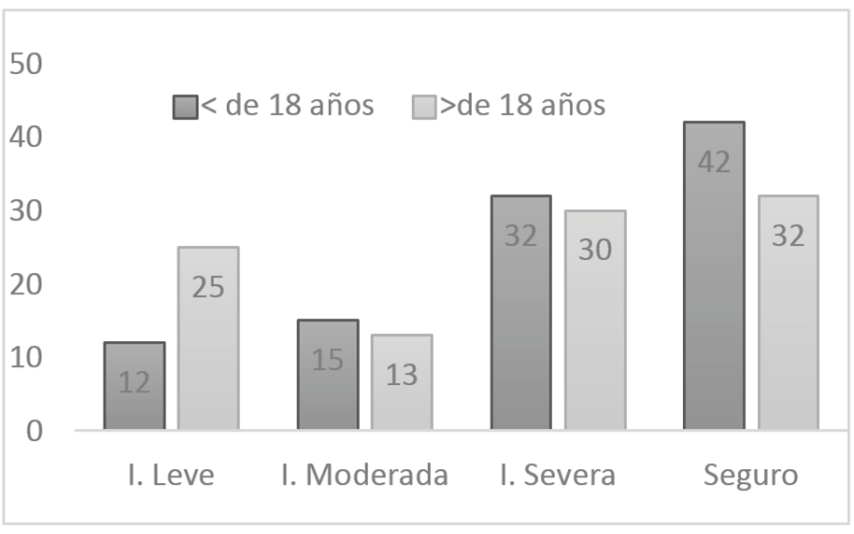

Figura 1. Prevalencia de inseguridad alimentaria en hogares. Kukra Hill RAACS, Nicaragua, 2015. Fuente: Encuesta Inseguridad Alimentaria en hogares.

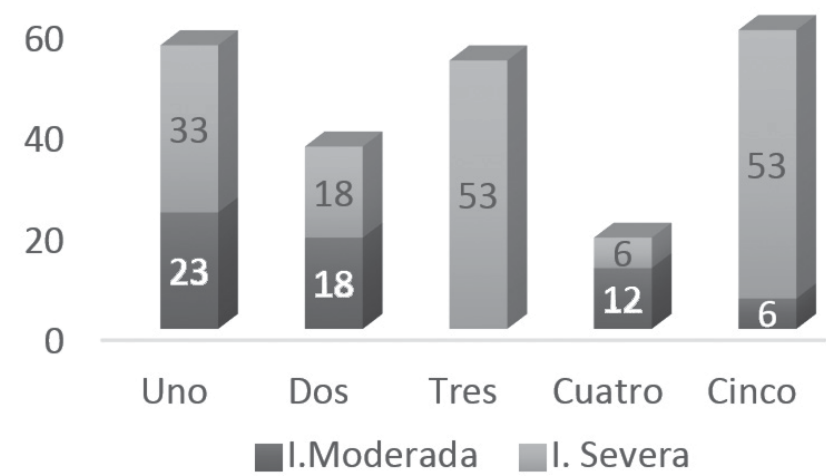

Figura 2. Porcentaje de inseguridad alimentaria nutricional moderada y severa en hogares según sector. Seguridad alimentaria y nutricional, Municipio de Kukra Hill, RACCS, Nicaragua, 2015. Fuente: Encuesta Inseguridad Alimentaria en hogares. Kukra Hill RACCS-Nicaragua 2015

Inseguridad alimentaria nutricional en hogares según trabajo. El resultado de la encuesta Inseguridad Alimentaria en hogares, Kukra Hill RACCS-Nicaragua 2015, muestra que el $30 \%$ de los hogares donde trabaja el jefe de familia hay Inseguridad Alimentaria Severa y un 10\% hay inseguridad moderada. Cuando el jefe de hogar no trabaja hay un $33 \%$ de Inseguridad Alimentaria Severa y un 20\% de Inseguridad Alimentaria Moderada. 
Inseguridad alimentaria nutricional moderada y severa según escolaridad del jefe de hogar. La Encuesta indica que los hogares cuyos jefes son analfabetos, tiene el 50\% de Inseguridad Alimentaria Severa, cuando lee y escribe presentan $25 \%$ la inseguridad severa; en cambio, los que alcanzan la primaria tienen $37 \%$ de inseguridad severa y $14 \%$ de inseguridad moderada. Con estudios en secundaria, la inseguridad alimentaria severa alcanza el 32\%. Cuando el jefe de hogar es profesional, la inseguridad alimentaria severa alcanzo un $12 \%$.

Inseguridad alimentaria nutricional moderada y severa según la cobertura del ingreso mensual. Los resultados de la encuesta muestran que los hogares cuyo ingreso cubre menos que el gasto mínimo tienen el 38\% de Inseguridad alimentaria severa y el 17 de inseguridad moderada. En los hogares cuyo ingreso cubre los gastos mínimos presentan el $35 \%$ de inseguridad alimentaria severa y el $8 \%$ de inseguridad moderada. En los hogares cuyo ingreso cubre más que el gasto mínimo, la inseguridad alimentaria severa alcanzo el $14 \%$ y la inseguridad alimentaria moderada alcanzo el 14\%.

Inseguridad alimentaria moderada y severa según Etnia. La encuesta indica que en los hogares cuyo jefe de hogar es de la etnia Miskito tiene el 63\% de Inseguridad Alimentaria Severa y el $13 \%$ de inseguridad alimentaria moderada. Cuando el jefe es de la etnia mestizo la inseguridad alimentaria nutricional severa alcanza el 30\% y la inseguridad moderada $14 \%$. Cuando el jefe de hogar es de la etnia Creole la inseguridad alimentaria severa alcanza el $25 \%$ y un $13 \%$ alcanza la inseguridad nutricional moderada.

Jefes de hogares preocupados porque los alimentos se acabaran en su hogar por falta de dinero u otros recursos. La encuesta Inseguridad Alimentaria en hogares, Kukra Hill RACCS-Nicaragua 2015, muestra que el 68\% de todos los jefes de hogares encuestados, mostro preocupación porque los alimentos se les acabaran.

Hogares que fueron afectados por la cantidad de alimentos, por falta de dinero u otros recursos. Los resultados muestran que en los hogares donde hay mayores de 18 años, fueron afectados por la cantidad limitada de comida, el $43 \%$ comió menos, un $41 \%$ recibió menos comida en su plato y el $47 \%$ dejo de desayunar, almorzar o cenar, por falta de dinero $\mathrm{u}$ otros recursos. En los hogares donde hay menores de 18 años también la cantidad de alimentos falto, el $47 \%$ comió menos, al $48 \%$ le disminuyeron la ración de comida y un $40 \%$ de ellos dejo de desayunar, almorzar o cenar por falta de dinero u otros recursos.

Hogares que fueron afectados por la calidad de alimentos, por falta de dinero u otros recursos. La encuesta indica que la calidad del alimento se presentó en todos los hogares encuestados. En los hogares con mayores de 18 años, el 53\% no comió comida saludable ni nutritiva, y un $40 \%$ tuvo poca variedad en los alimentos. En los hogares con menores de edad, un $47 \%$ no comió comida saludable ni nutritiva y el $51 \%$ no tuvieron variedad en los alimentos.

Hogares que fueron afectados por el Hambre, por falta de dinero u otros recursos. Según los resultados de la encuesta en todos los hogares encuestados el hambre en un momento les afecto, el $42 \%$ de los mayores de 18 años sintieron hambre, pero no tuvieron que comer, y un $42 \%$ comió sola una vez al día, o no comió nada durante todo el día. Afecto igual en los hogares con menores de 18 años, donde un 43\% sintió hambre y no había que comer, y un $43 \%$ comió una vez al día o no comió nada durante todo un día.

Más del 50\% de los jefes de hogares trabajan, sin embargo, tienen un ingreso que no alcanza el precio de la canasta básica, la mayoría tuvo un ingreso mensual menor a C\$ 6,500 córdobas y la canasta básica estaba en C\$12, 365.45. (INIDE, Diciembre 2015). Este ingreso limita el acceso seguro y permanente de los hogares, a la alimentación suficiente en cantidad y calidad. Los jefes de hogares no son pensionados, ni desempleados, ni dependientes de remesas, son personas que forman parte de la fuerza laboral permanente, con un ingreso que no permite el acceso, con familias extendidas en su mayoría, y único proveedor de su hogar. Esto los convierte en inseguros crónicos.

El hecho de que un adolescente reciba menos comida que lo que necesita, limita su crecimiento y desarrollo para una vida activa y saludable. Reducir el acceso a la cantidad y calidad de alimento está asociado a la obesidad. La falta de acceso trae consecuencias psicológicas graves por el aumento en el nivel de ansiedad y stress causado de la preocupación que el alimento se va a acabar o ya se acabó. Más del 50\% de los jefes de hogares alcanzaron una escolaridad menor a la secundaria, en donde el analfabetismo, y el saber y escribir tiene un peso significativo. La escolaridad es importante para obtener un trabajo mejor remunerado. Se corroboró que entre mayor el nivel educativo mayor la seguridad alimentaria y vice-versa.

De acuerdo con las estimaciones, existen alrededor de 400 grupos indígenas en América Latina que, según la fuente de información, representan entre 40 y 50 millones de personas (Stavenhagen, 1996). El conocimiento sobre estos pueblos, aunque fragmentado, muestra sostenidamente mayor incidencia de la pobreza, menor ingreso, escolaridad y esperanza de vida, mayor mortalidad infantil y materna, y menor acceso a la salubridad y al agua potable. Los pueblos indígenas de la región enfrentan una situación de exclusión y discriminación con relación a la población no indígena, lo que constituye un hecho inaceptable a la luz de los tratados internacionales. Este estudio refleja lo mismo toda la población Miskito encuestada refleja que son pobres, pues en la mayoría de los jefes de hogares expresaron que solo una per- 
sona aporta a la economía del hogar, más de la mitad no tiene casa, no tienen oportunidades para desarrollarse, tienen enfermos en casa, más de la mitad son analfabetos o solo saben leer y escribir. Más de la mitad de los que trabajan tienen un ingreso inferior a la canasta básica, y esto los hace ubicarse en el grupo de inseguridad alimentaria crónico.

Disponibilidad: la preocupación de los jefes de hogares que la comida se acabara y no contar con dinero u otros recursos, fue en la mayoría de los hogares, alcanzo hasta el $87 \%$ en los hogares cuyos jefes son Miskito. Cantidad de alimento, el alimento debe ser suficiente para cubrir las necesidades del organismo, los adolescentes tienen necesidades nutritivas marcadas por procesos de maduración sexual, aumento de talla y peso, aumento de masa corporal y aumento de masa ósea, por lo que requiere mayor cantidad de energía y nutrientes como carbohidratos, proteínas, grasas, vitaminas y minerales, en forma equilibrada o balanceada. En la mayoría de los hogares por falta de dinero u otros recursos, comieron menos de lo acostumbrado, y hasta dejaron de comer un tiempo de comida porque no había alimento en el hogar.

Calidad de alimento, el alimento debe ser completo, debe aportar todos los componentes necesarios para el organismo y que satisfaga la necesidad de comer, garantizando un buen estado de salud; desde el punto de vista fisiológico, psicológico y social. Debe ser equilibrado suficiente para cubrir las necesidades del organismo, adecuada, dependiendo de la edad, el sexo, las necesidades fisiológicas, de la estructura corporal y estado de salud. El rescate de los alimentos sanos y nutritivos es fundamental para mejorar la alimentación diaria y mejorar la calidad de dieta que nos ayude a tener una adecuada nutrición y salud. El tiempo de comida en los hogares no era completa, ni equilibrada, ni suficiente, ni adecuada en la mayoría de los casos. Estaba cargado de carbohidratos, falto una alimentación saludable y nutritiva en los hogares y hubo muy poca variedad.

Hambre es el mayor riesgo a la salud en el mundo. Es imprescindible dar prioridad a estas personas -especialmente las mujeres y los niños en cualquier etapa del ciclo biológicoy promover la aplicación de los principios de inclusión, igualdad, facilidad de acceso y transparencia. Al reducir el hambre se aumenta la productividad, ya que aumentan la capacidad de trabajo, el aprendizaje y el desarrollo cognitivo y se mejora igualmente la salud gracias a la menor incidencia de las enfermedades y la mortalidad prematura. El hambre y la mala salud afectan directamente a la creación de capital humano y social y al crecimiento económico, con unos efectos dura- deros y transgeneracionales que obstaculizan la consecución de otras metas sociales de alcance global. El hambre afecto a más del $40 \%$ de los hogares encuestados, manifestándose con los integrantes de los hogares que sintieron hambre pero no comieron, y comieron solamente un tiempo de comida y algunos no comieron durante todo el día alimentos por falta de alimentos u otros recursos para el acceso.

\section{CONCLUSIONES}

Los jefes de hogar proceden del Sector Geográfico 1, pertenecen a la religión católica, de escolaridad primaria, del sexo masculino, menores de 45 años, mestizos, viven en unión libre, trabajan, con un ingreso mensual menor a los C\$2,850 córdobas y de familia extendida de 4 a 8 miembros. La mayoría se consideran pobres, por no tener trabajo, no tener servicios públicos, no tener oportunidades de superación, no tener casa, no tener salud, no poder mantener a sus dependientes.

La Inseguridad Alimentaria Nutricional Severa se encontró en un tercio de los hogares encuestados. Esta se presentó en más de la mitad de los hogares de la etnia Miskito. Casi la mitad de los hogares presentaron dificultad en la disponibilidad de alimentos. En más de la mitad de los hogares encuestados alguna vez a algún adulto o menor de 18 años le sirvieron menos comida, comieron menos de lo acostumbrado, y por ultimo dejaron de desayunar, almorzar o cenar, por falta de dinero u otros recursos.

El acceso a los alimentos se manifestó a través de la calidad de los alimentos en los hogares. En más de la mitad de ellos, algún adulto mayor o algún menor de 18 años no tuvieron una alimentación saludable, solo carbohidratos, y hubo poca variedad en los alimentos que consumían. A pesar que la mayoría de los jefes de hogares están trabajando, el ingreso de la mayoría no alcanza ni para cubrir la tercera parte de la canasta básica.El hambre se sintió en todos los hogares, en más del $40 \%$ de ellos los adultos mayores como los menores de 18 años, sintieron hambre, pero no tuvieron alimentos para comer, y en ocasiones solo comían un tiempo de comida o pasaban todo el día sin comer.

Los factores que se encontró con asociación y que son estadísticamente significativas a la Inseguridad Alimentaria son: Ser de la etnia Miskita. (OR 4.68; $\left.\mathrm{Chi}^{2}=5.79\right)$. Vivir en la zona 3. (OR 3.43; $\mathrm{Chi}^{2}=5.03$ ); (Vivir en la zona 5. (OR 3.48; $\left.\mathrm{Chi}^{2}=5.64\right)$. Jefe de fam. Analfabeto. (OR 2.73; $\mathrm{Chi}^{2}=2.37$ ). Ingreso cubre menos de gastos. OR 2.05, $\mathrm{Chi}^{2}=3.43$ ). Considerarse pobre. (OR 1.97; $\left.\mathrm{Chi}^{2}=4.48\right)$. Tener Ingreso mensual menor que US \$ 100. (OR 2.56; $\left.\mathrm{Chi}^{2}=4.32\right)$

\section{REFERENCIAS BIBLIOGRÁFICAS}

FAO Comité Científico ELCSA (2012). Escala Latinoamericana y Caribeña de seguridad Alimentaria Nutricional:

Manual de uso y aplicación.

INIDE (Instituto Nacional de Información de Desarrollo, NI). 2008. Kukra Hill en cifras. 37 p. 2015. Anuario estadístico 2015. 410 p.

Stavenhagen, R. 1996. Los pueblos originarios: el debate necesario.176 p. 